04

\title{
Внутрирезонаторные голографические решетки и лазеры с управляемым спектром на их основе
}

\author{
() А.П. Погода ${ }^{1}$, В.М. Петров ${ }^{2}$, И.С. Хахалин ${ }^{1}$, Е.Э. Попов ${ }^{1,2}$, А.С. Борейшо ${ }^{1}$ \\ ${ }^{1}$ Балтийский государственный технический университет „ВОЕНМЕХ“ им. Д.Ф. Устинова, \\ 190005 Санкт-Петербург, Россия \\ ${ }^{2}$ Университет ИТМО, \\ 197101 Санкт-Петербург, Россия \\ e-mail: ap_pogoda@mail.ru
}

Поступила в редакцию 16.12.2020 г.

В окончательной редакции 16.12.2020 г.

Принята к публикации 22.12.2020 г.

Приведено сопоставление влияния внутрирезонаторных спектральных селекторов: статических решеток Брэгга и динамических решеток коэффициента усиления. Показано, что их влияние на спектральные свойства излучения лазера с петлевым резонатором аналогично. Проведенное статистическое исследование модового состава цугов импульсов генерации в режиме модуляции добротности позволяет сделать заключение о том, что перезапись решетки последующим импульсом цуга не препятствует излучению на той же частоте. Это открывает возможность частотной стабилизации излучения. Наличие внутрирезонаторной решетки Брэгга приводит к повышению вероятности сохранения частоты излучения в соседних импульсах цуга излучения. Показано, что наблюдается сужение спектра генерации импульса в цуге несмотря на то, что спектральная ширина решетки превышает спектральную ширину импульса генерации. Совместная работа статических и динамических внутрирезонаторных решеток позволяет создать линейку лазеров с высокой энергией в импульсе, качеством излучения, близким к дифракционному пределу, высокой пиковой мощностью и узким спектром излучения.

Ключевые слова: стационарная решетка, динамическая решетка, дифракционная эффективность, самонакачивающийся резонатор, обращение волнового фронта.

DOI: $10.21883 /$ OS.2021.04.50767.291-20

\section{Введение}

Объемные решетки Брэгга как спектральные селекторы излучения позволили решить задачу получения узкого спектра генерации лазеров, а также обеспечить перестройку длины волны излучения в рамках полосы усиления активных лазерных сред $[1,2]$. Наиболее широко данная идея получила развитие в области диодных и волоконных узкополосных лазеров, характеризующихся сравнительно низкой мощностью излучения [3,4]. Задачи дистанционного исследования и анализа состава газовых смесей, а также реализации нелинейных эффектов, чувствительных к спектральной яркости, диктуют необходимость разработки узкополосных лазеров с управляемым спектром средней и высокой мощности $[5,6]$.

Тем не менее ряд задач, связанных с недостатком мощности при воздействии узкополосного излучения, можно решить, повысив яркость лазера на определенной длине волны за счет подавления паразитных спектральных компонент излучения [7]. Использование внутрирезонаторных спектральных селекторов позволяет влиять на спектр излучения, выделяя определенные спектральные компоненты и повышая эффективность генерации в нужной части спектра излучения. В работе описан лазер, в конструкции которого совместно использованы стати- ческие отражательные решетки Брэгга и динамические решетки коэффициента усиления.

Ранее в работе [8] продемонстрирован ряд лазеров с внутрирезонаторной коррекцией искажений волнового фронта за счет процесса обращения волнового фронта (ОВФ) в результате четырехволнового смешения (ЧВС). Представлены одно-, двух- и трехпетлевые схемы ОВФ резонаторов с энергией в импульсе свободной генерации соответственно 550, 1100, $1250 \mathrm{~mJ}$ при энергии накачки $6.3 \mathrm{~J}$ и длительностью импульса накачки $475 \mu \mathrm{s}$. Режим пассивной модуляции добротности обеспечивался использованием пассивного насыщающегося поглотителя YAG : $\mathrm{Cr}^{4+}$ с начальным пропусканием $10 \%$, в результате чего наблюдался цуг импульсов с пиковой мощностью до $27 \mathrm{MW}$. Описан режим генерации одиночного импульса, полученный путем ограничения длительности импульса накачки. Параметр качества излучения во всех режимах $\mathrm{M}^{2}<1.2$. Также благодаря существенной спектральной селективности динамических объемных решеток коэффициента усиления и показателя преломления $[9,10]$ получено сужение спектра лазерного излучения в режиме генерации одиночного импульса. Ширина полосы в режиме генерации одиночного импульса не более $300 \mathrm{MHz}$, в режиме генерации цуга импульсов не более $1.2 \mathrm{GHz}$. Недостатком является нестабильность длины волны генерации от 

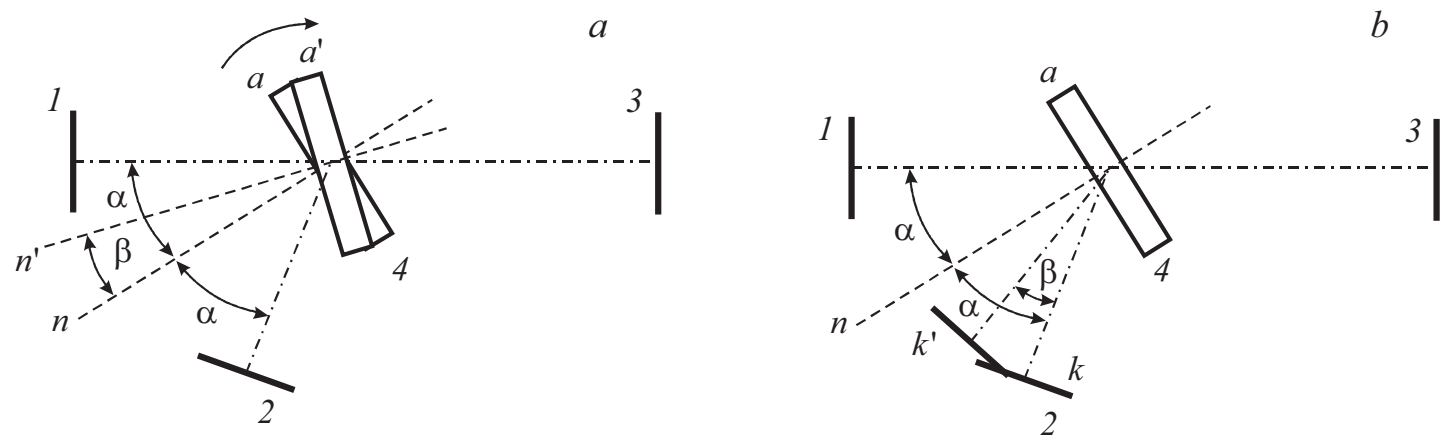

Рис. 1. Геометрия падения внутрирезонаторных лучей на решетку Брэгга: 1,2 - глухие зеркала; 3 - выходное зеркало; $4-$ образец с решеткой, $\alpha-$ угол Брэгга; $\beta$ - угол малого поворота; $n, n^{\prime}-$ нормаль к поверхности решетки Брэгга до поворота и после поворота; $k, k^{\prime}$ - угловое положение зеркала до поворота и после поворота. Угол падения и угол дифракции отстоят от угла Брэгга: $a$ - на равные углы (поворот решетки); $b-$ на неравные углы (поворот одного из зеркал).

импульса к импульсу. Актуальным является задача сохранения длины волны излучения в соседних импульсах цуга.

\section{1. Внутрирезонаторные голографические решетки}

Добротность резонатора с дифракционной решеткой Брэгга определяется дифракционной эффективностью решетки для каждой из спектральных компонент излучения, развивающегося в резонаторе. Рассмотрим случай строго монохроматического излучения с длиной волны $\lambda_{a}$. Решетка установлена таким образом, что угол падения луча совпадает с углом Брэгга для данной длины волны излучения. В результате дифракции на решетке направление распространения луча в резонаторе меняется. Для определенности будем называть один из лучей падающим на решетку, угол между ним и плоскостью решетки - углом падения, а луч, полученный в результате дифракции в первый порядок дифрагирующим, и соответственно угол с плоскостью решетки - углом дифракции. Таким образом, плоскость поверхности зеркала 1 строго перпендикулярна направлению распространения падающего луча, а зеркала 2 дифрагирующего луча. Очевидно, модовый состав лазерного излучения определяется в том числе угловой и спектральной селективностью решетки. Сочетание внутрирезонаторной решетки, конфигурации резонатора и активной среды позволяет осуществлять управление спектральными свойствами излучения. Рассмотрим влияние на спектр излучения поворота решетки, а также одного из двух зеркал. Схема резонатора приведена на рис. 1.

При повороте решетки Брэгга на угол $\beta$, угол падения, задаваемый перпендикуляром к поверхности зеркала 1 , составляет с нормалью к новому положению решетки угол $\alpha-\beta$. Угол дифракции, задаваемый положением зеркала 2 , составляет с этой нормалью угол $\alpha+\beta$. В результате из-за отклонения от угла Брэгга дифракционная эффективность решетки на данной длине волны падает. Уменьшение дифракционной эффективности одинаково в силу симметрии кривой ее зависимости от величины отклонения от угла Брэгга, так как величина модуля отклонения луча от оптимального одинакова. При этом фактически происходит разъюстировка резонатора и получают развитие лишь те длины волн, которые получают обратную связь от обоих зеркал 1 и 2. Следовательно, при увеличении угла поворота решетки интенсивность излучения, выходящего из резонатора с решеткой Брэгга, падает, а центральная длина волны контура остается постоянной и равной $\lambda_{a}$.

При повороте одного из двух зеркал, допустим зеркала 2 , на угол $\beta$, угол падения, задаваемый неизменным по положению зеркалом 1 , не изменяется. Угол дифракции, задаваемый повернутым зеркалом 2, составляет величину $\alpha-\beta$, т.е. имеет смещение, равное $\beta$ относительно угла Брэгга. Частота излучения, получающая наибольшую обратную связь от обоих зеркал, смещена относительно частоты, развивавшейся при начальном положении зеркала 2. В результате чего при повороте зеркала наблюдается смещение центральной частоты генерации лазера относительно длины волны $\lambda_{a}$ на величину, равную половине разницы между $\lambda_{a}$ и $\lambda_{a}+\Delta \lambda$, где $\lambda_{a}+\Delta \lambda-$ длина волны, для которой угол Брэгга данной решетки равен $\alpha-\beta$.

Таким образом, показано, что поворот внутрирезонаторной решетки в лазере приводит к падению интенсивности излучения, но не меняет центральную длину волны спектрального контура, тогда как поворот одного из внутрирезонаторных зеркал, напротив, приводит к смещению длины волны излучения в диапазоне контура усиления активной среды лазера.

Для исследования эффектов угловой и спектральной селективности динамических внутрирезонаторных решеток коэффициента усиления, сформированных в активной лазерной среде, возможно рассмотреть петлевой резонатор со статической решеткой Брэгга. Излучение, отклоненное в первый порядок дифракции 

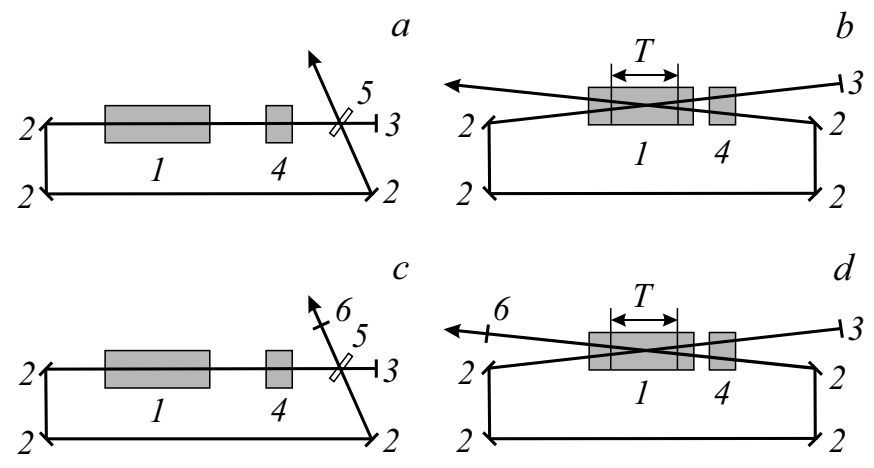

$e$

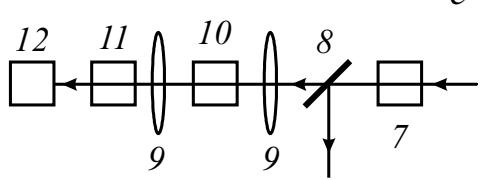

Рис. 2. Оптические схемы: резонаторов на основе стационарной брэгговской решетки $(a, b)$; на основе динамических решеток коэффициента усиления толщиной $T$, возникающих в объеме активного элемента $(c, d)$, с выходным зеркалом $(a, c)$ и без выходного зеркала $(b, d)$, измерения спектральных свойств излучения лазера $(e)$. На схеме: 1 - активный элемент $\mathrm{Nd}$ : YAG, 2 - зеркала, 4 - пассивный затвор YAG: $\mathrm{Cr}^{4+}$, 5 - решетка Брэгга, 6 - выходное зеркало. Оптическая схема измерительного стенда: 7 - генератор второй гармоники КТР, 8 - светоделитель, 9 - линзы, 10 - эталон Фабри-Перо, 11 - светофильтр, 12 - камера Ophir Spiricon.

решеткой Брэгга, формирует петлевой резонатор. Сопоставление влияния статических и динамических решеток на спектральные свойства лазерного излучения позволяет сделать выводы о характере угловой и спектральной селективности динамических решеток. Наличие выходного зеркала, формирующего дополнительную обратную связь, фиксирует пространственное положение петлевого резонатора, что оказывает особенно сильное влияние в случае динамических решеток. Таким образом, выходное зеркало также можно рассматривать как дополнительный угловой селектор излучения.

В ходе эксперимента производилось сравнение спектральных свойств излучения лазеров со следующими конфигурациями резонаторов. На схеме (рис. 2) активная среда $\mathrm{Nd}$ : YAG (1) помещалась в петлевой резонатор, сформированный зеркалами (2). Опорное глухое зеркало (3) обеспечивало достаточную для генерации добротность резонатора. Исследование проводилось в режиме модуляции добротности, который создавался как за счет пассивного затвора YAG : $\mathrm{Cr}^{4+}$ (4) с начальным пропусканием $10 \%$, так и за счет эффекта самомодуляции добротности в процессе формирования динамических решеток коэффициента усиления. Выходное зеркало (6) давало дополнительную обратную связь и фиксировало пространственное положение петлевого резонатора. На рис. 2, $a, b$ петлевой резонатор формируется статической решеткой Брэгга (5), а на рис. 2, $c, d$ динамическими решетками коэффициента усиления внутри активного элемента (1).

На рис. 2, e представлена схема измерения спектральных свойств излучения лазера. Пучок падал на интерферометр Фабри-Перо (10) с интервалом свободной дисперсии 27 pm, работающий в видимом диапазоне. Для преобразования излучения первой гармоники в видимый диапазон использовался генератор второй гармоники КТР (7), не преобразованное излучение отрезалось светофильтром (11). Фокусировка излучения осуществлялась линзами (9). Часть излучения отводилась светоделителем (8) для одновременной регистрации осциллограммы и интерферограммы излучения при помощи интерферометра Фабри-Перо. Регистрация интерферограмм производилась камерой Ophir Spiricon (12).

Измерения производились в режиме генерации цуга импульсов, содержащих от 1 до 5 продольных мод. При этом каждый импульс цуга является одномодовым, но наблюдается различие частоты генерации большинства импульсов цуга. Межмодовое расстояние составило $0.47 \mathrm{pm}$ в режиме второй гармоники. Соответствующее этому значению межмодовое расстояние первой гармоники $0.33 \mathrm{pm}$ позволяет рассчитать длину петлевого резонатора, которая составила $1.51 \mathrm{~m}$, что совпадает в пределах погрешности с длиной петли, реализованной в эксперименте.

Для исследования смещения частоты излучения в процессе генерации цугов импульсов рассматривалось независимо изменение частоты в результате вращения глухого зеркала, поворота решетки, а также, в случае наличия, выходного зеркала резонатора. На графике (рис. 3) показано смещение частоты в долях интервала свободной дисперсии эталона в зависимости от угла поворота глухого зеркала, предоставляющего обратную связь для излучения лазеров, реализованных по схемам, изображенным на рис. 2.

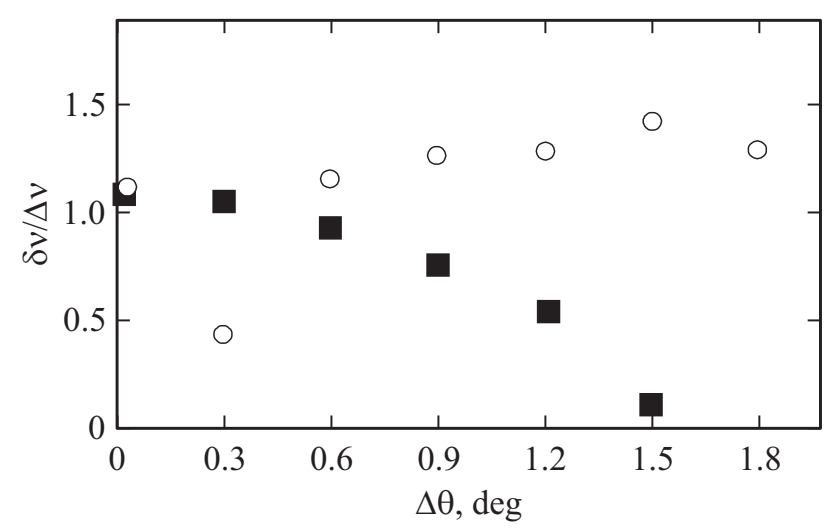

Рис. 3. Зависимость смещения частоты генерации, приведенная к ширине интервала свободной дисперсии. Квадраты схема с выходным зеркалом и статической решеткой (рис. 2,c), кружки - схема без выходного зеркала, но со статической решеткой (рис. 2, $a$ ). 
В случае отсутствия опорного зеркала и реализации петли за счет дифракции на стационарной решетке Брэгга (рис.2,a), рис. 3 (круги) демонстрирует хаотичное смещение частоты генерации в пределах $8 \mathrm{pm}$, несмотря на вращение опорного зеркала или решетки Брэгга. Данный спектральный диапазон соответствует ширине полосы генерации цуга импульсов. Это позволяет сделать вывод о том, что пространственное положение петли не фиксировано в пространстве и является уникальным для каждой частоты. В процессе развития генерации за счет конкуренции мод порог генерации преодолевает случайная частота этого диапазона. При этом положение центральной частоты не меняется, так как вращение опорного зеркала или решетки на угол $\alpha$ приводит к соответствующему смещению дифрагирующего луча.

Замена стационарной решетки Брэгга на динамическую решетку коэффициента усиления, которая записывается в активной среде интерферирующими внутрирезонаторными пучками (схема представлена на рис. $2, b$ ), приводит к аналогичному воздействию на спектр излучения. Вращение опорного зеркала за счет обеспечения обратной связи выделяет направление распространения внутрирезонаторных пучков, что приводит к повороту динамической решетки в пространстве. Диапазон угла поворота решетки задается апертурой торца активного элемента $(6.3 \mathrm{~mm})$. Период записанной решетки, определяющий ее спектральную и угловую селективность, остается прежним. Производится поворот петли резонатора в некотором диапазоне углов, соответственно положение центральной частоты остается постоянным, а смещение частоты происходит в диапазоне $7.5 \mathrm{pm}$. Соответствие значений центральной частоты и ширины диапазона смещения частоты генерации от импульса к импульсу позволяет сделать вывод о том, что динамические решетки коэффициента усиления действительно можно рассматривать как аналогичные решеткам Брэгга пространственные и спектральные селекторы частоты излучения.

Лазер с резонатором по схемам, реализованным согласно рис. 2, $c, d$, содержал дополнительное выходное зеркало с коэффициентом пропускания 60\%. Оно совместно с глухим зеркалом однозначно определяло направление оптической оси и строго фиксировало пространственное положение петли резонатора. Вращение зеркала, фиксирующего оптическую ось, производит отклонение падающего на решетку луча от угла Брэгга на величину $\beta$, тогда как положение дифрагирующего луча, задаваемое зеркалом, остается неизменным. В случае поворота описываемых зеркал наблюдается последовательное смещение центральной частоты генерации на величину $20 \mathrm{pm}$, что представлено на рис. 3 (квадраты). При этом смещение частоты от импульса к импульсу практически отсутствовало. Вращение решетки при сохранении положения петли резонатора приводит исключительно к падению спектральной яркости излучения. Так как падающий и дифрагирующий лучи при вращении решетки поворачиваются на одинаковый угол,

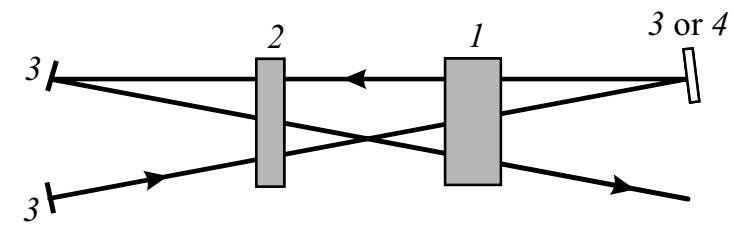

Рис. 4. Схема установки: 1 - активный элемент $\mathrm{Nd}: \mathrm{YAG}$, 2 - пассивный затвор YAG : $\mathrm{Cr}^{4+}, 3$ - зеркала, 4 - решетка Брэгга.

дифракционная эффективность решетки уменьшается. В результате при повороте решетки происходит смещение центральной частоты и падение спектральной яркости излучения.

В случае реализации петлевого резонатора по схеме, изображенной на рис. $2, d$, поворот одного из зеркал обратной связи относительно второго зеркала приводит к смещению нормали образуемой динамической решетки коэффициента усиления. Это приводит к упорядоченному смещению положения центральной частоты излучения, что особенно проявляется в режиме генерации цугов одиночных импульсов. При генерации цугов, состоящих из нескольких импульсов, наблюдается хаотичное колебание частоты вокруг положения центральной. Это объясняется тем, что конкурирующие моды петлевого резонатора занимают пространственные положения, смещенные на некоторый угол друг относительно друга. При этом глухое и выходное зеркала предоставляют неравномерную обратную связь для различных положений петлевого резонатора. В результате наблюдаются как упорядоченное смещение положения центральной частоты излучения, так и хаотичный сдвиг частоты от импульса к импульсу.

Проведенные сравнительные исследования позволяют заключить, что решетки коэффициента усиления, записанные в активной лазерной среде, оказывают эквивалентное статическим брэгговским решеткам воздействие на спектральные свойства излучения. Это позволяет использовать в отношении динамических решеток известные голографические соотношения угловой и спектральной селективности для толстых статических голограмм, а также производить таким образом расчет, позволяющий осуществлять управляемое воздействие на спектр излучения: перестройку длины волны и изменение ширины полосы генерации лазера.

\section{2. Сужение спектра генерации ОВФ-лазера решеткой Брэгга}

Одним из возможных путей стабилизации частоты генерации является использование дополнительного внутрирезонаторного спектрального селектора. Стабилизация частоты излучения производится в адаптивной лазерной системе с самонакачивающимся ОВФ с пассивным лазерным затвором (рис. 4) при четырехволновом 

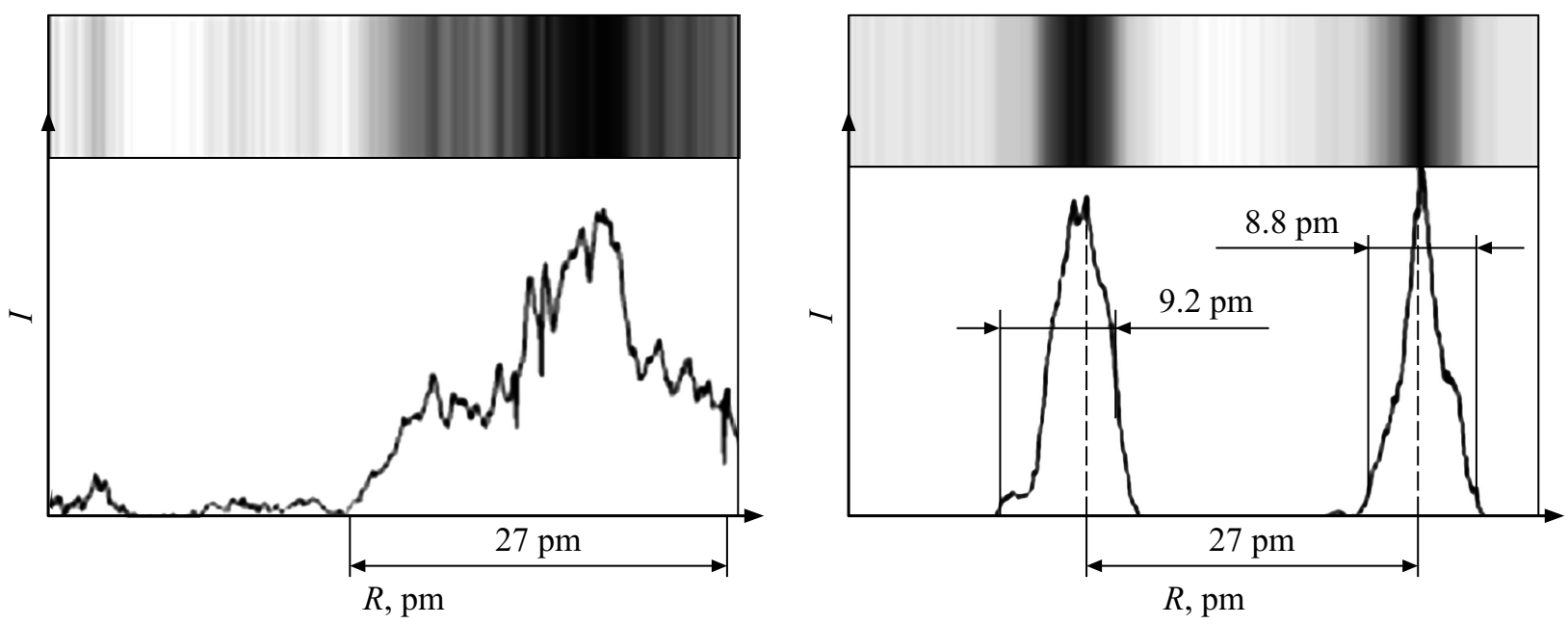

Рис. 5. Интерферограммы и их сечения: слева - без решетки Брэгга, справа - с решеткой Брэгга.

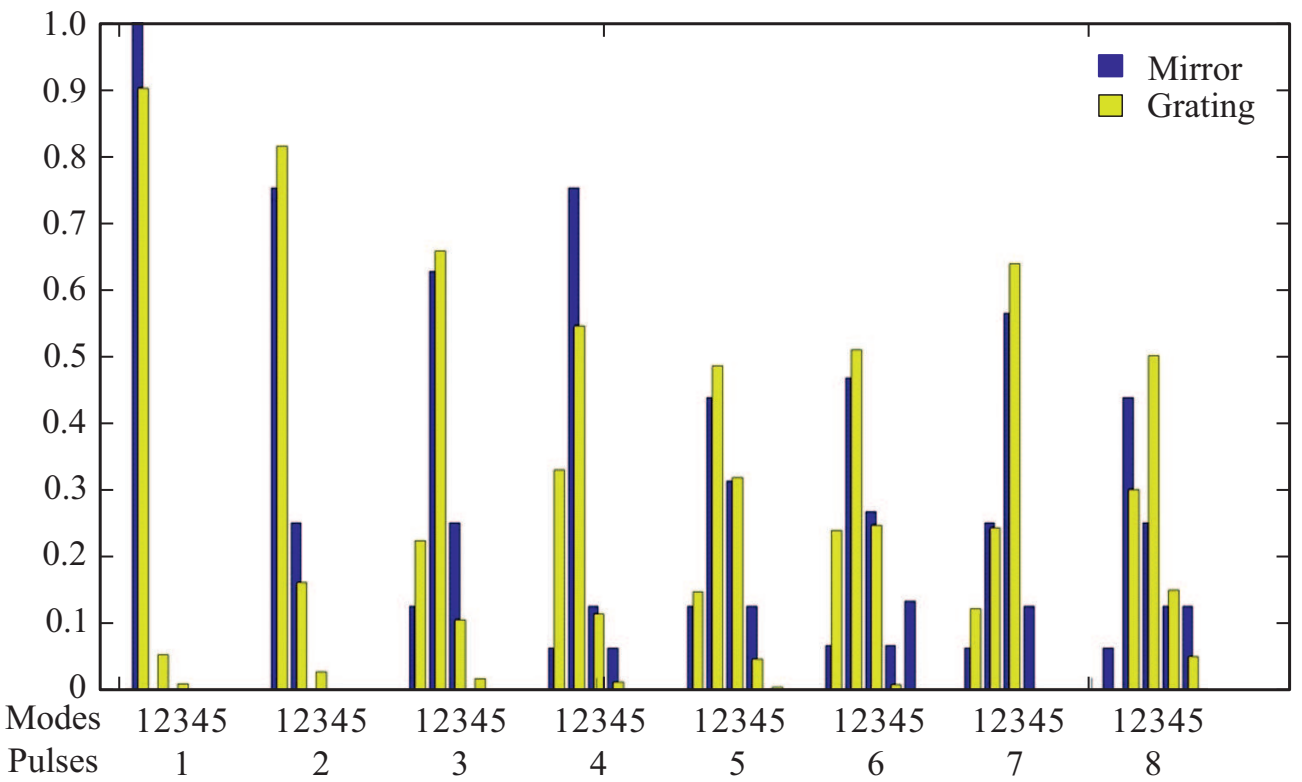

Рис. 6. Гистограмма вероятности генерации $m=1-5$ мод в цуге из $n=1-8$ импульсов в случае (желтый) с решеткой и (синий) без решетки.

взаимодействии в активном элементе $\mathrm{Nd}$ : YAG (1). В процессе развития генерации происходит включение динамического петлевого резонатора. Резонатор образован зеркалами (3). Максимальная энергия накачки составила 4 J. Пассивный затвор $\mathrm{YAG}: \mathrm{Cr}^{4+}$ (2) реализует режим пассивной модуляции добротности и позволяет получить излучение в виде цуга импульсов наносекундной длительности. Всравнительном эксперименте поворотное зеркало (3) заменялось отражательной решеткой Брэгга (4). Спектральная селективность решетки согласно паспорту составляет 50 pm. Проводилось сравнительное исследование спектральных свойств излучения лазера как в случае спектральной селекции с помощью отражательной решетки Брэгга, так и без нее. Решетка устанавливалась в резонатор вместо зеркала (3).
Лазерная генерация начинается с шумового излучения в активном элементе. По мере развития генерации внутрирезонаторными пересекающимися пучками в (1) записываются пропускающие решетки коэффициента усиления, образующие адаптивный резонатор. На образовавшихся динамических решетках происходит перераспределение поля излучения и обращение его волнового фронта. Решетка Брэгга выделяет центральные компоненты контура усиления, что приводит к стабилизации частоты генерации лазера. Записанные в активной среде динамические решетки в сочетании со стационарной решеткой Брэгга производят селекцию пространственных, спектральных и поляризационных характеристик. В результате дифракции на внутрирезонаторной решетке Брэгга когерентность излучения повышается с каждым 
проходом по резонатору. Это приводит к тому, что коэффициент обратной связи становится спектрально зависимым. Соответственно степень когерентности излучения и коэффициент обратной связи для каждой длины волны отличается. Он определяется степенью соответствия углу Брэгга внутрирезонаторной решетки. В результате введение малых дополнительных спектрально зависимых потерь приводит к существенному изменению спектра генерации.

На рис. 5 представлены примеры интерферограмм излучения лазера с резонатором без (слева) и с использованием отражательной решетки Брэгга (справа). Ширина спектра генерации уменьшилась с более чем $27 \mathrm{pm}$ до $9.2 \mathrm{pm}$.

Использование отражательной внутрирезонаторной решетки Брэгга позволяет снизить оптические потери по сравнению с использованием пропускающей решетки. При максимальной накачке в режиме пассивной модуляции добротности энергия излучения в цуге из 6 импульсов составляет величину $86 \mathrm{~mJ}$ и снижается до $78 \mathrm{~mJ}$ при использовании внутрирезонаторной решетки. Энергия в одном импульсе составляет $14 \mathrm{~mJ}$ и $13 \mathrm{~mJ}$ соответственно. Важно, что генерация лазера обладает высокой энергией в импульсе и пиковой мощностью излучения, является одномодовой и одночастотной, что делает лазер полезным для широкого круга практических задач в областях голографии, спектроскопии, генерации гармоник, прецизионной обработке материалов и других.

\section{3. Стабилизация частоты генерации ОВФ-лазера решеткой Брэгга}

В рамках исследования влияния решетки Брэгга в петле резонатора лазера с ОВФ производилась одновременная съемка интерферограмм и осциллограмм импульсов генерации в режиме модуляции добротности для двух конфигураций петлевого резонатора: без решетки Брэгга и с решеткой. Осуществлялась проверка гипотезы, что установка решетки Брэгга позволяет повысить стабильность генерации как вероятность сохранения частоты при генерации соседних импульсов цуга. На рис. 6 представлены полученные экспериментально вероятности генерации импульса и цугов импульсов с некоторым модовым составом. Группы столбцов обозначают количество импульсов в цуге, столбцы в группе обозначают модовый состав сгенерированного цуга импульсов. Подтверждением гипотезы о стабилизации являлось бы смещение центра масс распределений вероятности в каждой группе столбцов в сторону уменьшения количества мод - в левую сторону. По гистограмме видно, что в случае одного импульса генерации установка решетки приводит к генерации дополнительных мод. В случае двух импульсов в цуге видно, что решетка увеличила вероятность генерации одномодового цуга. В случае трех и более импульсов ситуация становится менее ясной, однако видно, что в подавляющем числе случаев вероятность генерации одномодового цуга возрастает.

\section{4. Заключение}

В работе показано, что решетка, помещенная внутрь резонатора, приводит к повышению спектральной яркости излучения лазера. Проведенные сравнительные исследования влияния статических решеток Брэгга и динамических решеток коэффициента усиления как элементов, образующих петлю резонатора, идентичны. Это позволяет использовать в отношении динамических решеток известные голографические соотношения угловой и спектральной селективности для толстых статических голограмм, а также осуществлять управляемое воздействие на спектр излучения: перестройку длины волны и изменение ширины полосы генерации лазера.

Излучение лазеров с многопетлевыми самонакачивающимися ОВФ-резонаторами, несмотря на высокую селекцию частоты за счет наличия динамических решеток коэффициента усиления, не является в полной мере одночастотным за счет изменения частоты излучения в диапазоне линии усиления. Проведенное статистическое исследование модового состава цугов импульсов в режиме модуляции добротности позволяет сделать заключение о том, что перезапись решетки последующим импульсом цуга не препятствует излучению на той же частоте. Этот вывод открывает возможность частотной стабилизации излучения. Наблюдается сужение спектра генерации импульса в цуге, несмотря на то, что спектральная ширина решетки существенно превышает спектральную ширину импульса генерации и даже линии усиления. Использование пропускающей или отражательной решетки Брэгга в резонаторе позволяет произвести существенное сужение спектра импульса генерации с более чем $27 \mathrm{pm}$ до $9.2 \mathrm{pm}$ при энергии в импульсе $13 \mathrm{~mJ}$.

\section{Финансирование работы}

Работа выполнена в организации ФГБОУ ВО БГТУ „ВОЕНМЕХ“ им. Д.Ф. Устинова при финансовой поддержке Министерства науки и высшего образования Российской Федерации (доп. соглашение от 09.06.2020 № 075-03-2020-045/2 на выполнение базовой части государственного задания „Разработка фундаментальных основ создания и управления группировками высокоскоростных беспилотных аппаратов космического и воздушного базирования и группами робототехнических комплексов наземного базирования“).

\section{Конфрликт интересов}

Авторы заявляют, что у них нет конфликта интересов. 


\section{Список литературы}

[1] Lumeau J., Glebov L.B., Smirnov V. // Opt. Lett. 2006. V. 31. N 16. P. 2417-2419.

[2] Pogoda A., Boreysho A. // Proc. SPIE. 2018. V. 11042. P. $110420 \mathrm{~K}$.

[3] Andrusyak O., Smirnov V., Venus G., Vorobiev N., Glebov L. // Proc. SPIE. 2009. V. 7195. P. 71951Q. https://doi.org/10.1117/12.813402

[4] Volodin B.L., Dolgy S.V., Melnik E.D., Downs E., Shaw J., Ban V.S. // Opt. Lett. 2004. V. 29. N 16. P. 1891-1893. https://doi.org/10.1364/OL.29.001891

[5] Boreysho A.S., Konyaev M.A., Morozov A.V., Pikulik A.V., Savin A.V., Trilis A.V., Chakchir S.Y., Boiko N.I., Vlasov Y.N., Nikitaev S.P., Rozhnov A.V. // Quantum Electronics. 2005. V. 35. N 12. P. 1167.

[6] Pogoda A.P., Lebedeva T., Yusupov M., Liventsov R., Lebedev V., Boreysho A., Gavrilov A., Smetanin S., Fedin A. // Proc. SPIE. 2013. V. 8677. P. $86770 Z$. https://doi.org/10.1117/12.2016458

[7] Pogoda A.P., Sergeev A.A., Khakhalin I.S., Popov E.E., Istomina N.L., Boreisho A.S., Petrov V.M. // Quantum Electronics. 2020. V. 50. N 7. P. 658-661.

[8] Погода А.П. // Автореф. канд. дис.: 01.04.03: СПб., 2015. $155 \mathrm{c}$.

[9] Eremeykin O.N., Antipov O.L., Minassian A., Damzen M.J. // Opt. Lett. 2004. V. 29. N 20. P. 2390-2392.

[10] Antipov O.L., Eremeykin O.N., Ievlev A.V., Savikin A.P. // Opt. Express. 2004. V. 12. P. 4313-4319. 